\title{
1 Smart Culture in Healthcare
}

\author{
Arno Elmer
}

Digitalisierung durchdringt alle Lebenswelten und Bevölkerungsgruppen. Industrie, Wirtschaft, Finanzen, Shopping, Kommunikation, Politik und Wahlen, Militär und Gesundheits- und Sozialwesen genauso wie jung und alt, reich und arm, 1., 2. und 3. Welt, gesund und krank. Und aus diesem Grund beschäftigen sich mittlerweile sehr viele, auch alle Wissenschaftsdisziplinen, mit diesem Thema: Ökonomie und Informatik, Medizin, Soziologie, Rechtswissenschaften und Ethik usw.

Digitalisierung soll „smart“ sein und daher wird dieser Begriff häufig in diesem Kontext verwendet. Smart als Synonym für einfach, clever und nützlich: Smart Home, Smart Living, Smart City, Smart Community, Smart Energy, Smart Education etc. Der Einfluss digitaler Instrumente, Devices, Apps, Sensoren, Plattformen, Datenbanken und Big Data, eCommerce-Prozesse bis hin zu künstlicher Intelligenz, Machine Learning, Smart Grid hat bereits zu einer starken Veränderung unseres Lebens, der Gesellschaft und auch von Moral, Werten und Kulturen geführt. Diese Entwicklung wird sich fortsetzen und weder vor Unternehmenskulturen noch vor dem Gesundheitswesen haltmachen.

Übrigens und an alle, die noch ernsthaft daran glauben oder darauf setzen, dass dieser Trend, dieser Hype wieder wegginge und man das nur aussitzen müsse: Die Erde ist keine Scheibe, Pferdekutschenwagen wurden (mit Ausnahme einiger Regionen in West-Utah) durch das sogenannte Automobil abgelöst, die Mauer ist gefallen, Quelle und Grundig sind pleite gegangen. 


\section{Digitalisierung geht nicht mehr weg! Oder noch etwas martialischer: Be fast or be food!}

Digitalisierung wartet nicht und wird schneller. Und da wir auf der einen Seite im Durchschnitt „durchschnittlich“ sind, werden viele „überdurchschnittlich“ IT- und Technik-affine Menschen diese Entwicklung offen, neugierig und positiv mitgehen und gestalten - viele Menschen aber eben auch nicht.

\section{Was ist Smart Culture?}

Wir entwickeln uns (oder vielmehr werden entwickelt) zur Smart Culture. Eine Kultur, die zu einem großen Anteil auf digital unterstützter oder vielmehr erst überhaupt ermöglichter Selbstbestimmung, Selbstdefinition und Freiheit basiert. Ein Freiheitsdrang, der gleichzeitig stückweise auch Sicherheit - weil seit Jahrhunderten aufgebaute Denk- und Verhaltensweisen sich durch Digitalisierung verändern - aufgibt. Smart Culture wird unterstützt von Techniken, die uns unglaublich schnell unfassbare Mengen an Informationen, Entscheidungshilfen und Kommunikationsmöglichkeiten bieten.

Durch die ständige digitale Verbundenheit, das Controlling und Monitoring durch Gadgets, moderne Technologien und Social Media Communities und Plattformen, kompensieren wir den Verlust der gewohnten, liebgewonnenen Sicherheit und erleben und gestalten selbst die neu erlangte Freiheit. Wir werden Smart. Unsere Intelligenz erlangt durch die Nutzung moderner Technologien eine nie dagewesene, rasend schnelle Umsetzung des Gedachten und unserer Vorstellungen. Unsere mentalen Kapazitäten werden durch die Nutzung moderner Medien und Tools um ein Vielfaches multipliziert. Unsere gestalterischen Fähig- und Fertigkeiten im Alltag, im Berufsleben, in der Freizeit und auch im Hinblick auf unsere Gesundheit haben ein Allzeithoch an Selbstverantwortung erlangt, die wir durch die Verwendung moderner Technologien und die fortschreitende Digitalisierung in der Lage sind, zu bewältigen - nicht immer, aber immer öfter.

\section{Technologischer Fortschritt}

Seit Beginn der Industrialisierung wird der technologische Fortschritt meist jubelnd vom Großteil der Menschheit begrüßt. Allerdings fast nie bereits schon bei der ersten Einführung neuer Techniken! Der technologische Wandel brachte und bringt Nutzen und gleichzeitig natürlich auch Veränderungen im Beruf, in der Bildung, in der Freizeit und im Alltag. Das Gesundheitswesen ist ebenfalls von diesen Veränderungen nicht ausgenommen und bahnbrechende Veränderungen und Erfindungen haben in Operationssäle, in Diagnoseverfahren und Behandlungsmethoden Einzug gehalten.

Digitale Instrumente und Prozesse sind schon Teil unseres Gesundheitswesens geworden. Dieses tut sich jedoch noch schwer damit, dem Patienten seinen wachsenden Wunsch nach Selbstbestimmung und Freiheit zuzugestehen. Unser Gesundheits- 
wesen ist (noch) nicht von Patient Enabeling und Empowerment geprägt, sondern meist von einer Arzt-Patienten-Beziehung, welche dem Patienten die Selbstverantwortung geradezu abspricht. Diese Beziehung ist in der heutigen Zeit unrealistisch und häufig auch ungesund. Als Menschen sind wir bereits Teil einer zunehmenden Smart Culture. Einer Gemeinschaft, die ihre wissenschaftlichen Leistungen und Errungenschaften sowie deren Fortentwicklung und Zukunft für alle digital verfügbar und zugreifbar macht. Diese Möglichkeiten existieren im Gesundheitswesen noch sehr eingeschränkt.

Die Umsetzung von Home Care Systems, Future Care Applikationen und E- und MHealth Anwendungen im Gesundheitswesen kann nur erfolgreich gelingen, wenn die Rolle des Patienten entsprechend modifiziert und vor allem in einer gestärkten und selbstverantwortlichen Form von Ärzten und Pflegepersonal akzeptiert wird. Selbstverwirklichung und Selbstverantwortung sind Kernaspekte der Smart Culture im Healthcare Bereich.

Mit jedem technologischen Fortschritt geht auch ein kultureller Wandel einher. Im Hinblick auf die Etablierung einer Smart Culture im Healthcare Bereich treibt die fortschreitende Digitalisierung diesen Wandel voran. Die Akzeptanz neuer technologischer Entwicklungen, ihre rasante Fortentwicklung sowie ihre immer weiter wachsende Präsenz in allen Aspekten unseres Lebens zeigt sich auch daran, dass sich die Kommunikationskultur in unserem Privatleben bereits gravierend verändert hat. Virtuelle Realitäten sowie der Einsatz von immer neuen Technologien stehen dabei erst noch am Anfang ihrer Entwicklung. Die reale Welt wird dabei nicht verdrängt, sondern immer mehr durch digitale, virtuelle Online-Welten erweitert. Der Mensch ist dabei Teil des digitalen Prozesses und der virtuellen Welten und wird Teilhaber an einer Smart Culture. In einer Kultur, in der er einen zunehmenden Teil seines persönlichen Lebensraums bereits mit digitalen Tools er- und begreift. Die tägliche Cestaltung des Arbeitsalltags ist bereits von Digitalisierung geprägt, aber auch in der Freizeit basiert vieles von unserem Wirken, Schaffen und Erleben auf digitalen Technologien.

\section{Hat der Transformationsprozess des Gesundheitswesens zu einer Smart Culture bereits begonnen?}

Die Antwort ist JA, denn Digitalisierung wartet und fragt nicht. Die Durchdringung der Lebenswelten mit neuen Kommunikationsmöglichkeiten und Techniken zur Erfassung, Verarbeitung und Analyse, der Speicherung und dem Austausch von Daten nimmt rasant zu. Diese wird auf Sektorengrenzen, föderale Zuständigkeiten, Grenzen zwischen Bezirken und Ländern, Rechtsräume wie SGB V und XI, Abgrenzungen zwischen Reha oder Medizin, Pflege oder private Helfer keine Rücksicht nehmen. Es geht nicht mehr darum, ob, sondern wie wir diesen Prozess gestalten. Die Gestaltung kann nur auf Basis von Freiwilligkeit und Unterstützung der „Willigen“ erfolgreich sein. Die Entwicklung einer dem Thema Digitalisierung positiv aufgeschlossenen Smart Culture, kann die evolutionäre Anpassung nachdrücklich begleiten. Es gilt, sich dabei natürlich auch mit den Risiken der Digitalisierung aktiv zu beschäftigen, ohne die nicht aufhaltsame Entwicklung einfach pauschal zu verdammen. 
Manager, Führungskräfte und Entscheidungsträger im Gesundheitswesen haben die Aufgabe, die notwendige kulturelle Weiterentwicklung der Unternehmen im Gesundheitswesen aktiv zu begleiten. Es müssen nachhaltige Kooperationen und Partnerschaften in Unternehmen und zwischen Institutionen formiert werden. So gilt es auch, eine Brücke zwischen dem 1. und dem 2. Gesundheitsmarkt zu etablieren. Die Gewinner werden nicht nur die Unternehmen sein, die das Thema aktiv gestalten, sondern vor allem deren Mitarbeiter, die sich durch adäquate Maßnahmen unterstützt und mitgenommen fühlen werden. 